\title{
Tumor-treating fields plus chemotherapy versus chemotherapy alone for glioblastoma at first recurrence: a post hoc analysis of the EF-14 trial
}

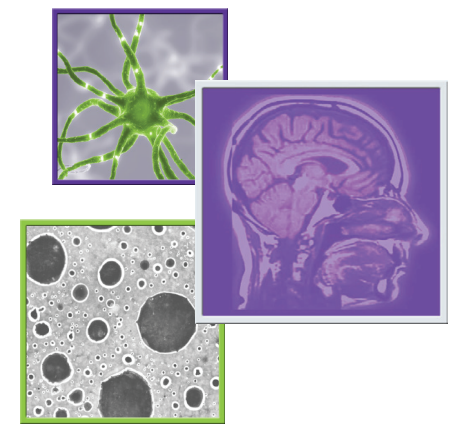

\author{
Santosh Kesari* ${ }^{* 1,2,3}$ \& Zvi Ram4; on behalf of EF-14 Trial Investigators
}

Background: This post hoc analysis of the EF-14 trial (NCT00916409) of tumor-treating fields (TTFields) plus temozolomide versus temozolomide alone in newly diagnosed glioblastoma compared the efficacy of TTFields plus chemotherapy (physician's choice) versus chemotherapy alone after first recurrence. Methods: Patients on TTFields plus temozolomide continued TTFields plus second-line chemotherapy after first recurrence. Some patients on temozolomide alone crossed over after approval of TTFields for recurrent GBM. The primary efficacy outcome was overall survival (OS). Results: After disease progression, 131 patients received TTFields plus chemotherapy and 73 chemotherapy alone. Thirteen patients in the original temozolomide-alone group crossed over to receive TTFields plus chemotherapy after disease progression, resulting in 144 patients receiving TTFields plus chemotherapy and 60 chemotherapy alone. Median follow-up was 12.6 months. Bevacizumab, alone or with cytotoxic chemotherapy, was the most frequent treatment. Median OS in the TTFields plus chemotherapy group was significantly longer versus chemotherapy alone (11.8 vs 9.2 months; HR: $0.70 ; 95 \% \mathrm{Cl}, 0.48-1.00 ; \mathrm{p}=0.049$ ). TTFields showed a low toxicity safety profile, as previously reported, with no grade $3 / 4$ device-related adverse events. Conclusion: TTFields plus chemotherapy after first disease recurrence on TTFields plus temozolomide or temozolomide alone prolonged OS in patients in the EF-14 trial.

First draft submitted: 29 December 2016; Accepted for publication: 6 March 2017; Published online: 12 April 2017

Glioblastoma (GBM) is the most common malignant tumor of the CNS; GBM accounts for $46 \%$ of primary malignant brain tumors [1], and its aggressive behavior presents unique treatment challenges. After maximal safe surgical resection, the conventional standard of care is radiotherapy with concomitant temozolomide followed by maintenance temozolomide [2]. Despite aggressive multimodal therapy, the prognosis of patients with GBM is poor; historically, the 2-year survival rate is $27 \%$, and only $10 \%$ of patients live longer than 5 years [3]. Therapeutic options are limited after progression following initial treatment, and clinical trials of investigational systemic therapies in the past decade have failed to improve outcomes of patients with GBM [4-8].

Tumor-treating fields (TTFields) is an approved treatment for patients with GBM that inhibits tumor cell growth by blocking cell division and replication. TTFields delivers low-intensity, intermediate-frequency $(200 \mathrm{kHz})$ alternating electric fields via transducer arrays applied to the shaved scalp [9-16]. In the randomized Phase III trial (EF-14; NCT00916409) of TTFields in newly

'Translational Neuro-Oncology Laboratories, UC San Diego, San Diego, CA, USA

${ }^{2}$ Department of Neurosciences, UC San Diego, San Diego, CA, USA

${ }^{3}$ Department of Translational Neurosciences \& Neurotherapeutics, Pacific Neuroscience Institute \& John Wayne Cancer Institute at

Providence Saint John's Health Center, Santa Monica, CA, USA

${ }^{4}$ Neurosurgery Department, The Tel Aviv Sourasky Medical Center \& Tel Aviv University Sackler School of Medicine, Tel Aviv, Israel

*Author for correspondence: Tel.. +1 310829 8265; Fax: +1 310582 7287; kesaris@jwci.org

\section{KEYWORDS}

- bevacizumab $\bullet$ disease recurrence $\bullet$ glioblastoma - overall survival

- second-line chemotherapy

- TTFields 
diagnosed patients with GBM after initial treatment with standard chemoradiation, the combination of TTFields plus maintenance temozolomide significantly improved progression-free survival (PFS) and overall survival (OS) compared with temozolomide alone in a prespecified interim analysis. Based on the observed survival benefits, the Independent Data Safety Monitoring Board recommended termination of the study [13]. TTFields is the first intervention in a decade to improve survival in patients with newly diagnosed GBM and was recently approved by the US FDA for use in combination with temozolomide [17].

GBM inevitably recurs, and new treatment options are needed to manage progressive disease. A previous randomized Phase III trial (EF11; NCT00379470) of TTFields monotherapy versus chemotherapy in patients with recurrent GBM did not show significant improvement in OS and PFS compared with chemotherapy; however, quality of life was improved in the TTFields group without the usual toxic effects associated with chemotherapy [12]. The real-world clinical practice setting of the Patient Registry Dataset (PRiDe) of 457 patients with recurrent GBM who received TTFields demonstrated significantly longer median OS compared with the EF-11 trial (9.6 vs 6.6 months; hazard ratio [HR]: 0.66; 95\% CI, 0.05-0.86; p = 0.0003) [18].

The objective of the current post hoc analysis was to evaluate the efficacy and safety of TTFields when added to second-line treatment according to physician's best choice after first disease recurrence among patients enrolled in the EF-14 Phase III trial.

\section{Materials \& methods}

\section{- Patient selection \& study design}

General details of this multicenter, open-label, randomized Phase III trial of TTFields plus temozolomide versus temozolomide alone in patients with newly diagnosed GBM have previously been reported by Stupp et al. [13]. Briefly, patients of $\geq 18$ years of age with histologically confirmed supratentorial GBM, who were progression free after having undergone maximal safe debulking surgery or biopsy, had completed standard fractionated radiotherapy plus concomitant temozolomide and had a Karnofsky performance status (KPS) score of $\geq 70 \%$ were enrolled. All patients provided written informed consent, and the institutional review boards or ethics committees of the 83 participating centers approved the study.
After completion of treatment with temozolomide and radiotherapy, patients were randomized (Figure 1) to receive maintenance temozolomide (150-200 $\mathrm{mg} / \mathrm{m}^{2}$ per day for 5 days, every 28 days for 6-12 cycles) [19] with or without TTFields, which was to be initiated within 4-7 weeks from the last dose of concomitant temozolomide and radiotherapy. Patients receiving TTFields were fitted with four transducer arrays placed on the shaved scalp and connected to a portable, battery- or power supply-operated device (Optune, NovoCure Ltd, Haifa, Israel) set to generate alternating electric fields of $200 \mathrm{kHz}$ within the brain. Transducer array placement was individualized to deliver optimal field intensity within the treated tumor as determined by a TTFields mapping software system (NovoTAL, NovoCure Ltd). After being trained to operate the device, the patient continued treatment at home. Transducer arrays were supplied in sterile packaging and replaced by the patient, a caregiver or a device technician twice per week. Short treatment breaks for personal care were allowed.

At first progression, second-line therapy was offered per local practice to patients who experienced tumor progression. Thirteen patients randomized into the temozolomide-alone group crossed over to receive TTFields plus secondline chemotherapy following commercial availability of TTFields for recurrent GBM after regulatory approval. Treatment with TTFields continued until the second radiologic progression or clinical deterioration, for a maximum of 24 months. The criteria for progression were based on the MacDonald criteria [20]: tumor growth of $>25 \%$ of the product of two perpendicular diameters compared with the smallest tumor area measured in the patient during the trial, and the appearance of one or more new tumors in the brain (diagnosed radiologically as GBM). The final determination of progression was made by independent radiology review in cases in which MRI was available. In cases in which MRI was not available, clinical progression was determined according to the following criteria: decline in functional status as indicated by a decrease of $\geq 20$ points in KPS, decline in neurologic function as indicated by a decrease of $\geq 2$ points in the Medical Research Council scale [21] or an increase of $\geq 50 \%$ in steroid dose.

\section{- Statistical analysis}

A post hoc analysis of OS, computed from the day of first progression until event (death) or censored 


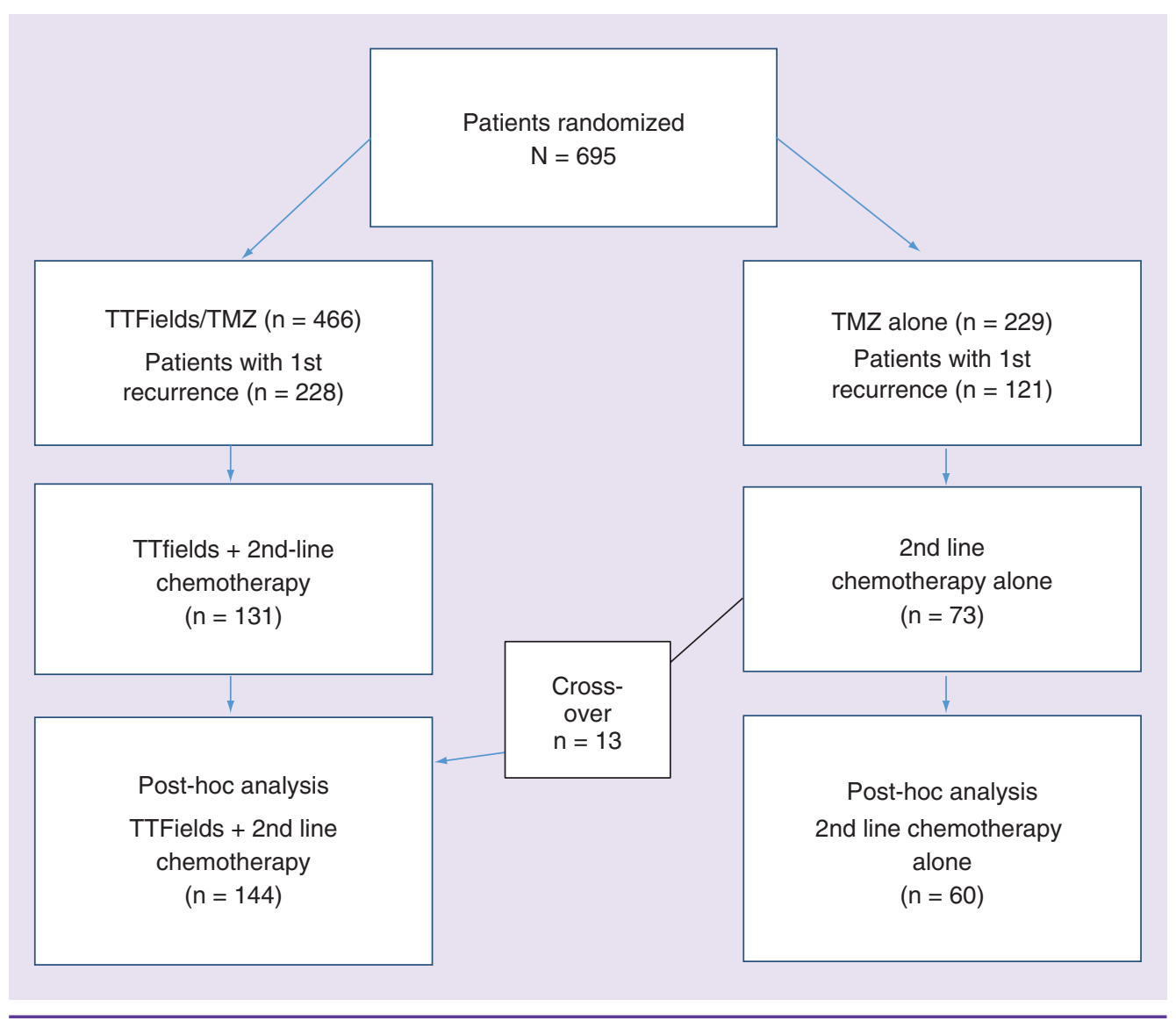

\section{Figure 1. Patient flow.}

TMZ: Temozolomide; TTFields: Tumor-treating fields.

at the last follow-up, was conducted according to the Kaplan-Meier method. Analyses were performed for all patients who progressed after any treatment. Time to first progression was based on the blinded central radiology review. All survival comparisons between groups were performed using a two-sided log-rank test with an $\alpha$-value of 0.05 . All statistical analyses were performed using SAS version 9.4 (SAS Institute, Inc., NC, USA).

\section{Results}

\section{- Patient characteristics}

A total of 695 patients with newly diagnosed GBM were randomized 2:1 to receive TTFields plus temozolomide $(n=466)$ or temozolomide alone $(n=229)$. Following temozolomide discontinuation due to toxicity or radiologic disease progression, second-line therapy (including reoperation, radiosurgery, chemotherapy, bevacizumab or combination therapy) was offered and continued until second progression for a maximum of 24 months. At the time of database lock (December 2014), 228 patients (48.9\%) in the
TTFields plus temozolomide group had a first recurrence, and 131 patients (28.1\%) received second-line chemotherapy in addition to continued TTFields treatment. In the temozolomidealone group, 121 patients $(52.8 \%)$ had a first recurrence and 73 patients (31.9\%) received second-line chemotherapy (Figure 1). Thirteen patients out of 73 in the temozolomide-alone group crossed over and received second-line therapy after disease progression in combination with TTFields after approval and commercial availability of TTFields for recurrent GBM. Therefore, 60 patients were treated with secondline chemotherapy alone, and 144 patients with TTFields plus second-line chemotherapy after first disease progression.

Patients' characteristics were well balanced between the two groups (Table 1). Median age was 57 years in the TTFields plus chemotherapy group and 58 years in the chemotherapy-alone group, and $75 \%$ of the patients in both groups were male. The median KPS score was $90 \%$ in both groups. About 58 and $57 \%$ of patients had gross total resection in the TTFields plus 
chemotherapy and chemotherapy-alone groups, respectively, and the frequency of MGMT methylation was 24 and $23 \%$.

\section{- Choice of second-line therapy}

Second-line treatments were well balanced between the TTFields plus chemotherapy and chemotherapy-alone groups. Bevacizumab (monotherapy or in combination with chemotherapy) was the most frequently used second-line treatment (53\% overall; $55 \%$ in the TTFields plus chemotherapy group; and 50\% in the chemotherapy-alone group; Table 2). Lomustine, carmustine and fotemustine were the most frequently used chemotherapy regimens, and were administered in 36 and 38\% of patients in the TTFields plus chemotherapy and chemotherapy-alone groups. Temozolomide treatment was continued in 17 and $12 \%$ of patients in the TTFields plus chemotherapy and chemotherapy-alone groups, respectively.

\section{- Efficacy}

After a median follow-up of 12.6 months, median OS in patients who received TTFields plus chemotherapy after first recurrence was 11.8 months, compared with 9.2 months in patients who received chemotherapy alone (HR: 0.70; 95\% CI, 0.48-1.00; p = 0.049; Figure $2 \mathrm{~A}$ ). As bevacizumab was the most frequent second-line treatment of choice, either as monotherapy or in combination with cytotoxic chemotherapy, OS was evaluated in the subset of patients who received bevacizumab with or without TTFields. Median OS from first disease recurrence with TTFields plus bevacizumab was 11.8 months, compared with 9.0 months in patients who received bevacizumab without TTFields treatment (HR: 0.61; 95\% CI, 0.37-0.99; $\mathrm{p}=0.043$; Figure 2B).

\section{- Safety}

Less than half of the patients treated with TTFields (49\%) experienced at least one grade $3 / 4$ treatment-emergent adverse event compared with $33 \%$ of patients who received chemotherapy alone (Table 3). Frequencies of thrombocytopenia, convulsion, hemiparesis, headache and mental status changes were numerically higher in the TTFields plus chemotherapy group compared with the chemotherapy-alone group. Medical device site reaction was reported in 19 patients (13\%) who received treatment with TTFields, and none was severe. Epilepsy was reported in three patients $(2 \%)$ in the TTFields plus chemotherapy group and two patients (3\%) in the chemotherapy-alone group; no grade $3 / 4$ seizures were reported in either group.

\section{Discussion}

GBM is a highly aggressive brain tumor with a poor prognosis, and TTFields is the first major treatment advance in this patient population over the past decade. TTFields was approved by the FDA as monotherapy for the treatment of recurrent GBM based on results from the EF-11

Table 1. Baseline characteristics.

\begin{tabular}{|c|c|c|}
\hline Characteristic & $\begin{array}{l}\text { TTFields plus second-line } \\
\text { chemotherapy }(n=144)\end{array}$ & $\begin{array}{l}\text { Second-line chemotherapy } \\
\text { alone }(n=60)\end{array}$ \\
\hline Median age, years (range) & $57(29-83)$ & $58(22-75)$ \\
\hline \multicolumn{3}{|l|}{ Sex, n (\%): } \\
\hline- Male & $108(75)$ & $45(75)$ \\
\hline - Female & $36(25)$ & $15(25)$ \\
\hline $\begin{array}{l}\text { Median Karnofsky performance status } \\
\text { score (\%) (range) }\end{array}$ & $90(60-100)$ & $90(70-100)$ \\
\hline \multicolumn{3}{|c|}{ Extent of resection at the time of diagnosis, $\mathrm{n}(\%)$ : } \\
\hline - Biopsy & $20(14)$ & $10(17)$ \\
\hline - Gross total resection & $84(58)$ & $34(57)$ \\
\hline - Partial resection & $40(28)$ & $16(27)$ \\
\hline \multicolumn{3}{|l|}{ MGMT status, $\mathrm{n}(\%):$} \\
\hline - Methylated & $35(24)$ & $14(23)$ \\
\hline - Unmethylated & $59(41)$ & $25(42)$ \\
\hline - Invalid & $21(15)$ & $10(17)$ \\
\hline - Unknown & $29(20)$ & $11(18)$ \\
\hline
\end{tabular}


Table 2. Type of second-line chemotherapy after first recurrence.

\begin{tabular}{|lll|}
\hline Second-line chemotherapy & $\begin{array}{l}\text { TTFields plus second-line } \\
\text { chemotherapy }(\mathbf{n}=144) ; \mathbf{n}(\%)\end{array}$ & $\begin{array}{l}\text { Second-line chemotherapy } \\
\text { alone }(\mathbf{n}=60) ; \mathbf{n}(\%)\end{array}$ \\
$\begin{array}{l}\text { Bevacizumab: } \\
\text { - Monotherapy }\end{array}$ & $61(42)$ & $26(43)$ \\
- Combination therapy & $18(13)$ & $4(7)$ \\
Lomustine, carmustine, fotemustine & $52(36)$ & $23(38)$ \\
\hline Temozolomide & $25(17)$ & $7(12)$ \\
\hline Irinotecan & $3(2)$ & $2(3)$ \\
\hline Carboplatin & $3(2)$ & $1(2)$ \\
\hline Procarbazine & 0 & $1(2)$ \\
\hline TTFields: Tumor-treating fields. & & \\
\hline
\end{tabular}

trial [12]. A prespecified interim analysis in the EF-14 trial demonstrated a significant improvement in median PFS (7.1 vs 4.0 months) and OS (20.5 vs 15.6 months) in patients receiving TTFields plus standard maintenance temozolomide versus temozolomide alone [13]. Based on these positive results, enrollment in the trial was terminated, and TTFields was approved by the FDA for the treatment of adult patients with newly diagnosed GBM [17]. This post hoc analysis from the interim analysis population of the EF-14 trial demonstrated that TTFields significantly improves OS when combined with second-line treatment after first disease recurrence. NCCN guidelines recommend systemic chemotherapy and/or bevacizumab for the treatment of recurrence [22] and this analysis showed that continued treatment with TTFields in addition to secondline treatment significantly improved OS in this patient population. The results suggest that the OS benefit from adding TTFields to second-line treatment may be independent of the concurrent systemic therapy. However, it should be acknowledged that this study provides no information on the possible benefit of continuation of TTFields alone in this patient population.

The survival durations of the chemotherapy group in our analysis are comparable with the previously reported data. For example, the reported median OS in a pivotal trial of bevacizumab for recurrent GBM ranged from 8.7 to 9.2 months [23], and a recent analysis of realworld data demonstrated median OS approaching 9 months with a bevacizumab-containing treatment in bevacizumab-naive patients initiating second-line therapy for recurrent GBM [24]. In a retrospective analysis of fotemustine as second-line treatment for recurrent GBM in elderly patients, the median OS from initiation of therapy was 7.1 months [25]. The addition of TTFields to bevacizumab or other second-line therapy in the current report further extends median OS by 2 months beyond that achieved with secondline treatment alone, and supports earlier results from the PRiDe real-world study of the safety and efficacy of T'TFields (used as monotherapy or in combination with systemic treatment[s]) in patients with recurrent GBM [18]. A third of the patients from PRiDe were treated with TTFields after a first recurrence and achieved a median OS of 20 months. This was significantly longer than that in patients treated at their second recurrence ( 8.5 months) or at their third or subsequent recurrence ( 4.9 months). In addition, the PRiDe data established the importance of treatment compliance for extending OS with TTFields (a median OS of 13.5 months in patients with $\geq 75 \%$ daily compliance with device use vs 4.0 months in patients with $<75 \%$ daily compliance), without any new safety signals compared with EF-11. Taken in conjunction with the previously reported benefits with TTFields for newly diagnosed GBM in the EF-14 interim analysis [13], our results support the early initiation and continued use of TTFields in combination with standard systemic therapies for the treatment of GBM.

The toxicity profile was similar in patients treated with TTFields plus chemotherapy after first recurrence compared with patients treated with TTFields plus temozolomide as maintenance therapy [13]. A numerically higher incidence of adverse events in the TTFields group could have been a consequence of the longer duration of follow-up compared with the patients treated with chemotherapy alone. Notably, there were no reported incidences of grade $3 / 4$ seizure in either group in the current study. In addition, only $13 \%$ of patients treated with TTFields reported a medical device site reaction, and none 
was severe. Patients usually tolerate the Optune device well, without adverse effects on quality of life, cognitive or functional capabilities with equal cognitive, emotional, physical and social functioning scores, and superior global health status ratings to the control arm demonstrated in the EF-14 study [26,27]. These data support the continued use of TTFields plus chemotherapy after first recurrence.

Prospective trials are currently underway to evaluate the administration of TTFields plus bevacizumab to patients with GBM, including in patients with newly diagnosed unresectable GBM in combination with temozolomide (NCT0234359), in patients with recurrent GBM (NCT01894061 and NCT02663271) and in patients with bevacizumab-refractory recurrent GBM (NCT02743078). These studies will provide additional insight into the efficacy and safety of TTFields in combination with bevacizumab as first-line and second-line therapy for GBM. In addition, ongoing analyses

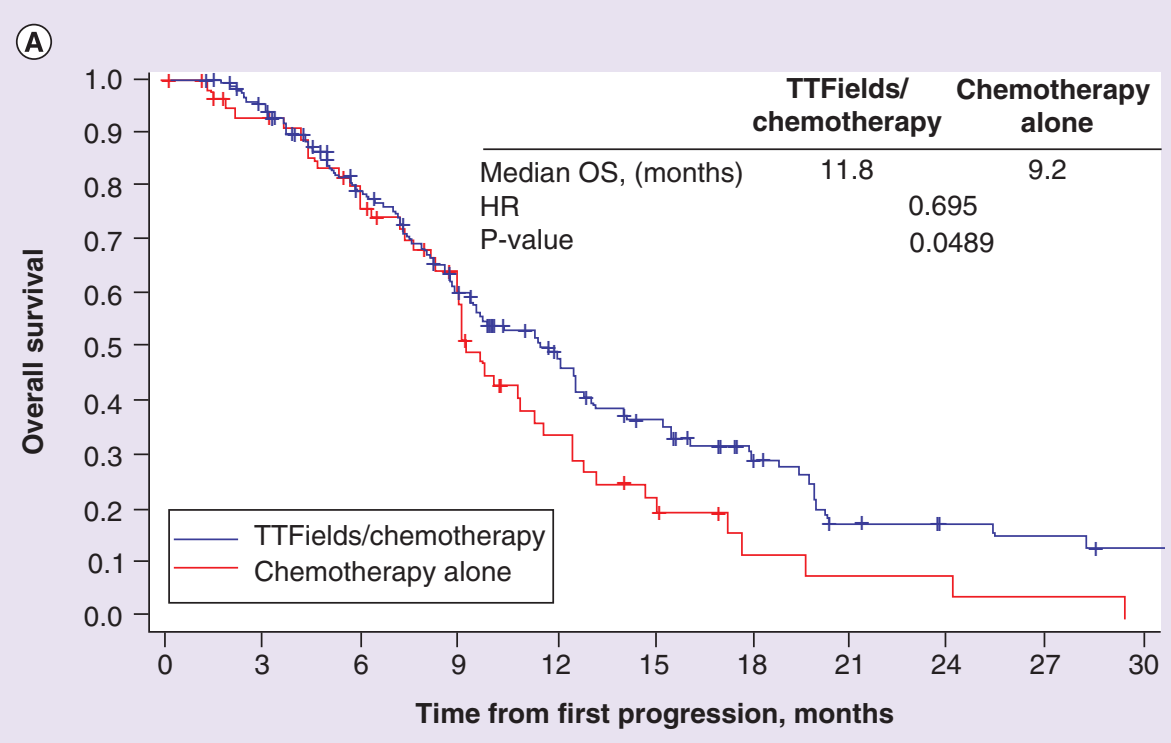

$\begin{array}{ccccccccccc}144 & 130 & 99 & 69 & 46 & 33 & 21 & 11 & 8 & 7 & 7 \\ 60 & 52 & 41 & 27 & 15 & 9 & 3 & 2 & 2 & 1 & 0\end{array}$

(B)

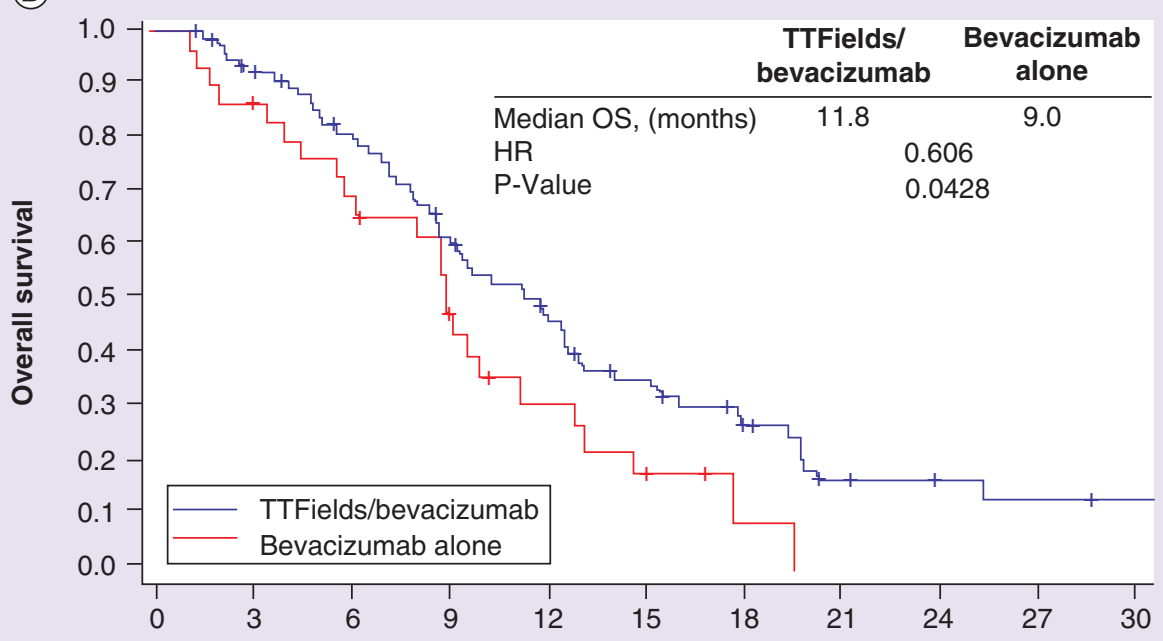

Figure 2. Overall survival from first disease recurrence. (A) TTFields plus second-line chemotherapy versus second-line chemotherapy alone, or (B) TTFields plus bevacizumab alone or in combination with chemotherapy versus bevacizumab alone or in combination with chemotherapy. HR: Hazard ratio; OS: Overall survival; TTFields: Tumor-treating fields. 


\section{CLINICAL TRIAL EVALUATION}

Table 3. Most common grade $3 / 4$ treatment-emergent adverse events ( $\geq 2 \%$ in the tumor-treating fields plus second-line chemotherapy arm).

\begin{tabular}{|c|c|c|}
\hline System organ class/preferred term & $\begin{array}{l}\text { TTFields plus second-line chemotherapy } \\
(\mathrm{n}=144) ; \mathrm{n}(\%)\end{array}$ & $\begin{array}{l}\text { Second-line chemotherapy alone } \\
(n=60) ; n(\%)\end{array}$ \\
\hline Patients with $\geq 1$ grade $3 / 4$ adverse event & $70(49)$ & $20(33)$ \\
\hline Blood and lymphatic system disorders: & $16(11)$ & $2(3)$ \\
\hline - Thrombocytopenia & $10(7)$ & $1(2)$ \\
\hline - Lymphopenia & $6(4)$ & $1(2)$ \\
\hline - Leukopenia & $4(3)$ & 0 \\
\hline Gastrointestinal disorders & $5(3)$ & 0 \\
\hline General disorders and administration site conditions: & $12(8)$ & $4(7)$ \\
\hline - Fatigue & $5(3)$ & $2(3)$ \\
\hline - Gait disturbance & $3(2)$ & $1(2)$ \\
\hline Infections and infestations: & $9(6)$ & 0 \\
\hline - Meningitis & $3(2)$ & 0 \\
\hline Injury, poisoning and procedural complications: & $7(5)$ & $2(3)$ \\
\hline - Fall & $4(3)$ & 0 \\
\hline Metabolism and nutrition disorders & $4(3)$ & $4(7)$ \\
\hline Musculoskeletal and connective tissue disorders & $5(3)$ & $2(3)$ \\
\hline Nervous system disorders: & $40(28)$ & $11(18)$ \\
\hline - Convulsion & $8(6)$ & $2(3)$ \\
\hline - Hemiparesis & $6(4)$ & 0 \\
\hline - Headache & $5(3)$ & $2(3)$ \\
\hline - Cognitive disorder & $4(3)$ & $1(2)$ \\
\hline - Neurological decompensation & $3(2)$ & $1(2)$ \\
\hline Psychiatric disorders: & $6(4)$ & $1(2)$ \\
\hline - Mental status changes & $4(3)$ & 0 \\
\hline Vascular disorders & $5(3)$ & $1(2)$ \\
\hline TTFields: Tumor-treating fields. & & \\
\hline
\end{tabular}

of biomarker data from the EF-14 trial may help identify the subset(s) of patients most likely to benefit from continued TTFields treatment in combination with standard of care.

It is important to note that these results are limited by the post hoc nature of the analyses and by the fact that the second-line TTFields group was heterogeneous for prior treatment history (i.e., included a small subset of patients who had initiated TTFields at first recurrence in addition to a larger group of patients who had received TTFields upfront). Moreover, most of the patients who continued TTFields after recurrence had relapsed after first-line TTFields therapy, and the choice of second-line systemic therapy was based on local practice; these factors may have had confounding effects. Nonetheless, the concordance in median OS results between the chemotherapy groups in our analysis and earlier reports [23-25] supports the validity of these results. Overall, our report supports the continued use of TTFields in patients with GBM after first disease recurrence.

\section{Conclusion}

This post hoc analysis suggests that treatment with TTFields improves OS when combined with second-line treatment after first disease recurrence. Continued treatment with TTFields in addition to second-line treatment with bevacizumab with or without concomitant chemotherapy also significantly improved OS, indicating that the OS benefit from adding TTFields to second-line treatment may be independent of the systemic therapy being used. It is important to note that these results are limited by the post hoc nature of the analyses and by the fact that the second-line TTFields group included a small proportion of patients who did not receive TTFields as initial therapy. Overall, our report supports the continued use of TTFields in patients with GBM after first disease recurrence.

\section{Acknowledgements}

The authors thank the patients and their families for participating in the trial. They are grateful to all of the EF-14 


\section{CLINICAL TRIAL EVALUATION}

investigators and the nursing staff for taking care of the patients.

\section{Disclosure}

Portions of the data were presented at the 2015 Society for Neuro-Oncology Annual Meeting (abstract ATNT-19).

Financial \& competing interests disclosure

The study was funded by NovoCure Ltd. S Kesari is a consultant with Novocure and has received research grants from the company. Z Ram is a consultant and shareholder with Novocure and has received research grants from the company. The authors have no other relevant affiliations or financial involvement with any organization or entity with a financial interest in or financial conflict with the subject matter or materials discussed in the manuscript apart from those disclosed.

No writing assistance was utilized in the production of this manuscript.

\section{EXECUTIVE SUMMARY}

\section{Background}

- Tumor-treating fields (TTFields) is an approved treatment for adults with glioblastoma (GBM), combined with temozolomide as maintenance treatment in newly diagnosed patients and as monotherapy for recurrent disease.

- TTFields delivers low-intensity, intermediate-frequency alternating electric fields that inhibit tumor growth by blocking cell division and replication.

\section{Design of study}

- The EF-14 trial in patients with newly diagnosed GBM showed that the combination of TTFields and maintenance temozolomide (after initial chemoradiation) significantly increases progression-free survival and overall survival (OS) compared with maintenance temozolomide alone.

- This post hoc analysis was designed to evaluate the efficacy and safety of TTFields combined with second-line therapy after first recurrence in patients included in EF-14.

\section{Key results}

- After first progression, 131 patients in the original TTFields plus temozolomide arm continued with TTFields and a further 13 patients in the temozolomide-alone arm crossed over to TTFields combined with physician's choice of second-line therapy. Sixty patients in the temozolomide-alone arm received second-line systemic therapy alone after progression.

- Second-line treatments were similar between the two groups, with bevacizumab alone or in combination the most frequent choice.

- After a median follow-up of 12.6 months, the median OS was 11.8 months in the patients who received TTFields plus chemotherapy compared with 9.2 months in those who received chemotherapy alone (HR: $0.70 ; 95 \% \mathrm{Cl}: 0.48-1.00$; $\mathrm{p}=0.049$ ).

- Treatment with TTFields was well tolerated with a similar toxicity profile to that previously reported.

\section{Conclusion \& clinical significance}

- This post hoc analysis showed that TTFields combined with second-line treatment significantly increased OS after first recurrence of GBM.

- The benefit of TTFields appeared independently of the second-line therapy used.

- The results support the continued use of TTFields after first relapse.

\section{References}

Papers of special note have been highlighted as:

- of interest; $\bullet$ of considerable interest

1 Ostrom QT, Gittleman H, Fulop J et al. CBTRUS statistical report: primary brain and central nervous system tumors diagnosed in the United States in 2008-
2012. Neuro Oncol. 17(Suppl. 4), iv1-iv62 (2015).

2 Gallego O. Nonsurgical treatment of recurrent glioblastoma. Curr. Oncol. 22, e273-e281 (2015).

3 Stupp R, Hegi ME, Mason WP et al. Effects of radiotherapy with concomitant and adjuvant temozolomide versus radiotherapy alone on survival in glioblastoma in a randomised Phase III study: 5 year analysis of the EORTC-NCIC trial. Lancet Oncol. 10, 459-466 (2009).

4 Gilbert MR, Wang M, Aldape KD et al. Dose-dense temozolomide for newly diagnosed 
glioblastoma: a randomized Phase III clinical trial. J. Clin. Oncol. 31, 4085-4091 (2013).

5 Gilbert MR, Dignam JJ, Armstrong TS et al. A randomized trial of bevacizumab for newly diagnosed glioblastoma. N. Engl. J. Med. 370, 699-708 (2014).

6 Chinot OL, Wick W, Mason W et al. Bevacizumab plus radiotherapy-temozolomide for newly diagnosed glioblastoma. N. Engl. J. Med. 370, 709-722 (2014).

7 Stupp R, Hegi ME, Gorlia T et al. Cilengitide combined with standard treatment for patients with newly diagnosed glioblastoma with methylated MGMT promoter (CENTRIC EORTC 2607122072 study): a multicentre, randomised, open-label, Phase III trial. Lancet Oncol. 15, 1100-1108 (2014).

8 Westphal M, Heese O, Steinbach JP et al. A randomised, open label Phase III trial with nimotuzumab, an anti-epidermal growth factor receptor monoclonal antibody in the treatment of newly diagnosed adult glioblastoma. Eur. J. Cancer 51, 522-532 (2015).

9 Kirson ED, Dbaly V, Tovarys F et al. Alternating electric fields arrest cell proliferation in animal tumor models and human brain tumors. Proc. Natl Acad. Sci. USA 104, 10152-10157 (2007).

- Study of the effects of tumor-treating fields (TTFields) on living tissues, including cultured cell lines and animal tumor models.

10 Kirson ED, Schneiderman RS, Dbaly V et al. Chemotherapeutic treatment efficacy and sensitivity are increased by adjuvant alternating electric fields (TTFields). BMC Med. Phys. 9, 1 (2009).

- In vitro and animal studies showing that TTFields increase the efficacy of chemotherapy without increasing toxicity.

11 Fonkem E, Wong ET. NovoTTF-100A: a new treatment modality for recurrent glioblastoma. Expert Rev. Neurother. 12, 895-899 (2012).

- Review of TTFields as a treatment for glioblastoma.

12 Stupp R, Wong ET, Kanner AA et al. NovoTTF-100A versus physician's choice chemotherapy in recurrent glioblastoma: a randomised Phase III trial of a novel treatment modality. Eur. J. Cancer 48, 2192-2202 (2012).

- Phase III trial showing that the efficacy of TTFields is comparable with chemotherapy for the treatment of recurrent glioblastoma; toxicity and quality of life results favored TTFields.

13 Stupp R, Taillibert S, Kanner AA et al. Maintenance therapy with tumor-treating fields plus temozolomide vs temozolomide alone for glioblastoma: a randomized clinical trial. JAMA 314, 2535-2543 (2015).

-. Interim analysis of Phase III trial showing that adding TTFields to maintenance of temozolomide treatment for newly diagnosed glioblastoma (after initial chemoradiation) significantly prolonged progression-free and overall survival.

14 Kim EH, Kim YH, Song HS et al. Biological effect of an alternating electric field on cell proliferation and synergistic antimitotic effect in combination with ionizing radiation. Oncotarget 7(38), 62267-62279 (2016).

- In vitro study suggesting that TTFields and radiation have synergistic effects in suppressing cell migration and invasion.

$15 \mathrm{Kim}$ EH, Song HS, Yoo SH et al. Tumor treating fields inhibit glioblastoma cell migration, invasion and angiogenesis. Oncotarget 7(40), 65125-65136 (2016).

- In vitro study of the molecular mechanisms of tumor cell inhibitions, showing that TTFields inhibit glioblastoma cell invasion, migration and angiogenesis.

16 Clark PA, Gaal JT, Strebe JK et al. The effects of tumor treating fields and temozolomide in MGMT expressing and non-expressing patient-derived glioblastoma cells. J. Clin. Neurosci. 36, 120-124 (2017).

17 US Food \& Drug Administration. FDA approves expanded indication for medical device to treat a form of brain cancer (2015). www.fda.gov/NewsEvents/Newsroom

18 Mrugala MM, Engelhard HH, Dinh Tran D et al. Clinical practice experience with
NovoTTF-100A system for glioblastoma: The Patient Registry Dataset (PRiDe). Semin. Oncol. b41(Suppl. 6), S4-S13 (2014).

19 Stupp R, Mason WP, van den Bent MJ et al. Radiotherapy plus concomitant and adjuvant temozolomide for glioblastoma. N. Engl. J. Med. 352, 987-996 (2005).

20 Macdonald DR, Cascino TL et al., Schold SC Jr Response criteria for Phase II studies of supratentorial malignant glioma. J. Clin. Oncol. 8, 1277-1280 (1990).

21 Medical Research Council Brain Tumour Working Party. Prognostic factors for high-grade malignant glioma: development of a prognostic index. A Report of the Medical Research Council Brain Tumour Working Party. J. Neurooncol. 9, 47-55 (1990).

22 NCCN Clinical Practice Guidelines in Oncology - central nervous system cancers version 1.2016. www.nccn.org/professionals/physician_gls

23 Friedman HS, Prados MD, Wen PY et al. Bevacizumab alone and in combination with irinotecan in recurrent glioblastoma. J. Clin. Oncol. 27, 4733-4740 (2009).

24 Chen C, Ravelo A, Yu E et al. Clinical outcomes with bevacizumab-containing and non-bevacizumab-containing regimens in patients with recurrent glioblastoma from US community practices. J. Neurooncol. 122, 595-605 (2015).

25 Santoni M, Scoccianti S, Lolli I et al. Efficacy and safety of second-line fotemustine in elderly patients with recurrent glioblastoma. J. Neurooncol. 113, 397-401 (2013).

26 Zhu JJ, Pannullo S, Mehdorn M et al. ACTC-35, quality of life, cognitive function and functional status in the EF-14 trial. Neuro Oncol. 17, v1-v9 (2015).

27 Hottinger AF, Pacheco P, Stupp R. Tumor treating fields: a novel treatment modality and its use in brain tumors. Neuro Oncol. 18(10), 1338-1349 (2016).

- Comprehensive review of the mechanism of action, preclinical and clinical data and future potential of TTFields. 